Purdue University Purdue e-Pubs

\title{
Understanding Library Users' Preferences and Expectations of Online Help
}

Tao Zhang

Purdue University, zhangtao2000@gmail.com

Ilana Stonebraker

Purdue University, stonebraker@purdue.edu

Marlen Promann

Purdue University,mpromann@purdue.edu

Follow this and additional works at: http://docs.lib.purdue.edu/lib_fsdocs

\section{Recommended Citation}

Zhang, Tao, Ilana Stonebraker and Marlen Promanm. "Understanding library users' preferences and expectations of online help." Reference Services Review 44.3 (2016): 362-374.

This document has been made available through Purdue e-Pubs, a service of the Purdue University Libraries. Please contact epubs@purdue.edu for additional information. 


\title{
Understanding Library Users' Preferences and Expectations of Online Help
}

\author{
[Author information removed for blind review.]
}

\section{Structured Abstract}

Purpose: Online help and tutorials are an important part of library services, yet they are often studied in specific contexts and disciplines such as subject-specific research guides. The objective of this study was to examine users' common preferences and expectations of library help channels in general and online help in particular.

Methodology: We conducted a qualitative survey with 45 library users. The survey asked users how they seek library help, their preferences and expectations of online help, content format, and general help channels. We performed content analysis of survey responses.

Findings: Results showed that survey participants have different prioritizations of library help channels. Half of the respondents preferred conceptual help that emphasizes concepts and underlying principles, while the other half preferred procedural (step-bystep) help or mixed. The survey also pointed to reliance by participants on expert help, even when online help was available.

Value: Based on the results, we identified users' behavioral preferences, attitudes, and expectations towards library help channels and online help content. We also discussed the unique challenge of creating online help for libraries, as users have a dynamic range of help seeking preferences and mixed expectations of help content depending on the context. 


\section{Introduction}

Online help and tutorials are interactive, library-produced help systems with both static and dynamic content, as well as asynchronous and real-time communication channels between users and service providers. Over the last decade, online help has proliferated across libraries. For example, LibGuides, a popular help documentation platform (Springshare n.d.), powers over 430,000 guides across 66,000 libraries worldwide. Help documents like LibGuides are an important part of a library's online service for users to learn how to effectively use library resources.

Despite the advancement of technology, users may still encounter difficulties in their searching and information-seeking process with the library resources (e.g., discovery tools, catalogs, databases, e-books, etc.). For example, they may not understand the differences between databases and discovery tools; they may not be familiar with library jargon; and certain databases (e.g., geographic information systems (GIS)) may require orientation for users without relevant background. In these situations, users often turn to librarian-curated online help systems, which may include library guides, tutorials, screencasts, or digital reference.

Previous studies (e.g., Trenner 1989) have shown that online help systems were often inadequately designed and did not offer effective assistance to novice and infrequent users, which in turn lead to users' unwillingness to use online help systems. To address this issue, online help should not be assembled just to make it available to users. A well-conceived online help should meet users' preferences and expectations of their help seeking. While much assessment has been done of online help from the content 
usage standpoint, there have been fewer studies examining users' preferences and needs of online help.

In this study, we explore and qualitatively analyze important determinants of help seeking behavior for library resources, including users' preferences of different help channels, users' perceived importance of online help in the library context, users' preferences of help content format and features, as well as their general preferences of help channels.

Help design principles and guidelines are seemingly dispersed in the literature and sometimes inconsistent; therefore, it is necessary to establish empirical evidence from actual users to better inform online help design and integration with other library help channels. It is our intent not to prove that users prefer one system over another, but to understand more deeply how help-seeking preferences affect format preferences and vice versa. Understanding the different types of user preferences sheds light on the success, failure, and peaceful cohabitation of different help systems, which may appeal to different types of users.

\section{Related Work}

The library is still the first choice of study environment for students on campus to do study and research, as they need "a sort of universal service point, a physical Google" (Foster \& Gibbons 2007, p.76). Because of the need for a centralized place to start, libraries usually offer multiple help channels for users, including face-to-face help desk, online chat, email, text messaging, and online help documentation such as guides, frequently asked questions (FAQs), and tutorials. The availability of other channels may 
affect whether and when users choose to use online help. Previous surveys indicated that the majority of undergraduate students prefer face-to-face help in the libraries over virtual references like online chat, and that graduates working outside the library may have higher tendency to use online help (Granfield \& Robertson 2008). Another survey of undergraduate students found that respondents had a relatively strong preference for seeking help at a location that is convenient to them (not necessarily at the library). Respondents rated email as the most preferred channel to seek help rather than chat or texting, course page, and social network sites like Facebook and Myspace (Ismail 2010).

There have been studies on help-seeking behavior within various library contexts, such as online collaborative group work (Du et al. 2015), adult social work students (Ismail 2013), and mobile applications (Tsai et al. 2015). Personal and contextual factor could influence a user's decision to use help (Fisher 1983). Librarians have undertaken various studies to evaluate the effective of digital reference services (Pellegrino 2012; Luo 2014). While these studies are useful to libraries looking for best practices in designing online help, they focus on the behaviors of students and effectiveness of existing systems, versus examining how the behaviors of students are reflected in users' preferences in online library help systems.

The design of online library help could benefit from research findings on technical help documentation. Both library and technical help systems provide users with information to identify problems, solve problems, and perform tasks correctly. Regarding help documentation, Novick and Ward (2006) conducted in-depth individual interviews with 25 professionals using computer applications. They found that users generally preferred help documentation that is easy to navigate, provides explanations at an 
appropriate level of technical detail, enables finding as well as solving problems through examples and scenarios, and is complete and correct. These preferences require a careful balance between coverage and precision of help content, as well as variations of technical complexity of explanation for different users. The study also suggested that professionals were generally reluctant to experiment and explore to solve their problems in using an application, possibly due to the perceived value of their time. Novick and Ward provided important themes for the design of help documentation, but their study was focused on professionals using business applications, which could be different from library users seeking help for using scholarly resources.

Studies ononline technical help have explored user preferences of specific help formats. For example, Purchase and Worrill (2002) empirically tested and ranked a set of online help features (e.g., index, balloon help, and graphical examples) and design principles (e.g., "the help system should be unobtrusive" and "help should be context sensitive"). They suggested that design improvements of help features could lead to major usability improvements of online help systems. However, the majority of design principles were based on a high level of abstraction, making them prone to individual interpretation and lack of design consistency in practice. It is thus important to develop guidelines for online help that are more specific to the library context.

In the library context, Iris Xie and colleagues have done a number of studies on users' help-seeking behavior with help systems in digital libraries. Xie and Cool (2007) reviewed users' requirements on help features, including search query examples, demos, context-sensitive help, tutorials, and FAQs. They demonstrated that users prefer examples and step-by-step instructions as the least effort principle prescribes. Users also 
require help information to be specific, visual, and interactive. Xie (2007) characterized the help features of six digital libraries, including types (explicit versus implicit), formats (texts, images, screenshots, multimedia materials, and interactive formats), and presentation styles (descriptive, guided, procedural, and exemplary). There could be correlations between these features. For example, guided styles were correlated to formats such as screenshots, multimedia materials, and interactive formats, while procedural styles were frequently applied in FAQs. Moreover, there could be trade-offs between explicit and implicit help, and general versus specific help, in order to provide help information for different levels of users and help all users achieve their task goals. These trade-offs were similar to Novick and Ward's discussion about the balance between coverage and precision, as well as variations of technical complexity.

The format of online help could affect users' learning performance, although there are mixed results from previous studies. Craig and Friehs (2013) compared two common types of online information literacy tutorials for undergraduate biology lab class: a video tutorial with animation and narration and a HTML-based tutorial with static images. The results showed that students in the video tutorial condition had higher scores on quiz questions and more confidence after learning than the HTML condition. On the contrary, Mestre (2012) found that students performed better after viewing static web page tutorial than they did after viewing a screencast tutorial. Students preferred the HTML tutorial because it was easier to skim or jump to the sections they needed.

In summary, our review of previous research on online help and design principles shows that there is still a need to better understand users' preferences of library help in general and online help in the library context in particular. With the majority of online 
help supporting libraries' reference service, it is difficult to predict when and how users may encounter difficulties that require them to use the online help. Users often use the library resources during their study or research activities, making it challenging to plan meaningful observations in a limited time period. Therefore, in this study we chose the survey method to uncover user preferences and expectations, which are significant contributors to user satisfaction of online library help. The survey results may be less reliable than participatory evaluation and direct observation (Novick et al. 2007), but the resulted user preferences and expectations could help designers and content creators develop better understanding of the design space and explore new content strategies.

\section{Method}

Forty-five participants ( 21 females and 24 males, mean age is 23.1 years and standard deviation is 7.9 years) were recruited online and randomly in the library space to answer a survey about their experience, preferences and expectations of library help. The majority of participants $(39,86.7 \%)$ are undergraduate students, and the rest are graduate students and faculty. The detailed breakdown of participants' academic status is shown in Table 1. Participants' major ranged from Engineering, Science, Liberal Arts and History, to Arts and Humanities. All participants reported familiarity of searching on the library website and using the online library help.

\section{[Insert Table 1 about here.]}

The survey took place in a computer lab inside the library space. Upon arrival, participants first signed a consent form and completed a demographic and background questionnaire regarding their experience of using the library website and online help. 
Participants were then instructed to open the survey using the computer in front of them and provided written answers to the questions in the survey. None of the questions in the survey were mandatory. The average time for participants to complete the survey was about 20 minutes. Participants were given lunch sandwiches after they completed the survey as a small token of appreciation.

The survey consisted of the following behavioral and attitudinal questions:

(1) What is your usual way to get help using library resources? (Ask at the desk? Ask your class instructor or another student? Use online help? Experiment on your own? Or other ways? Tell a recent story or give an example.)

(2) In a "perfect library" or ideal situation other than what you have experienced, how would you get help?

(3) If online help is developed to a point that would allow you to learn to use the library website efficiently, would you be inclined to use the help? If not, could you explain why?

(4) When you get help, do you like it to be procedural (step-by-step) or do you prefer it be more conceptual (diagrams, concept maps)? What do you look for when you read help pages?

(5) What are the channels you prefer to get help information from? (For example, do you like to be able to chat or talk to someone right then when you are confused, or do you prefer to read something static like Wikipedia? Other options include chat, phone, email, static web page, etc.)

Questions 1 and 2 were to discover how users are currently using the available help channels from the library and their expectations of library help channels. Question 3 
asked users' preferences of using online help and the importance of online help in their help seeking routine. Question 4 tapped into users' preferences of specific formats for common library help content and their expectations of different help features. Question 5 examined users' general help seeking preferences and whether the library context affected their help seeking behavior. To give participants a more specific context and help them better understand the questions, we provided examples or prompts for Questions 1, 4, and 5. Those examples were not close-ended choices that participants had to select for their responses. We encouraged participants to respond to the questions exactly as how they would like to answer them in a face-to-face interview. We also asked participants to explain their initial answers and tell stories from their past experiences.

\section{Results}

Two researchers independently conducted an initial analysis of participant responses to the survey questions. We first identified common themes (i.e., categories) from the responses and then assigned categorical codes to each response. The initial analysis had $70 \%$ agreement on the categories and $75 \%$ agreement on the code assignment. The two researchers reviewed each other's initial analysis result, and then discussed the categories and codes assigned for each response. The final categories and code assignment reached $100 \%$ consensus. Many responses were assigned with multiple categorical codes. In addition, the researchers identified potential connections between responses to different survey questions. A sample of the survey responses is shown in Appendix B.

The descriptive statistics for the coded responses to Question 1 are listed in Table 2. Asking the library help desk, experimenting, and searching the library website are the 
top three most frequent choices for participants, while a smaller number of responses mentioned library FAQs or tutorials, Google, and asking a particular librarian. In addition to individual channels, one prominent pattern emerged from the responses: when participants had difficulties using the library resources, they would first experiment on their own and then ask the library help desk, another peer user, or an instructor if they could not solve the problem. A less prominent pattern is searching the library website or doing general web search using Google.

[Insert Table 2 about here.]

Table 3 shows the distribution of codes for responses to Question 2 (ideal way of getting help). Similarly to Question 1, the frequent channels for participants to find help include asking a librarian at a reference desk, help from librarian or expert (not necessarily at reference desk), and finding information on their own. Less frequent channels mentioned in the responses include online chat or text messaging, online help portal, centralized search, etc. Some of the responses are essentially participants' requirements of help information or service from the library, including physical closeness, availability, efficiency, ease of use, and clarity of information.

[Insert Table 3 about here.]

The majority of respondents $(32,71.1 \%)$ expressed willingness to use online library help mainly because of the advantages of online help information: efficiency to learn, convenience, easy to access, and availability. The rest of participants were inclined to one-and-one personal help or had mixed preferences (Table 4).

[Insert Table 4 about here.] 
Regarding the question of whether respondents prefer procedural (step-by-step) or conceptual (i.e., diagram, concept map, etc.) help information, relatively more respondents $(21,46.7 \%)$ preferred procedural help and $11(24.4 \%)$ respondents preferred conceptual help (Table 5). As the frequencies of personal preferences in Table 5 show, personal preference is the most significant factor influencing the responses. Participants first answered their preferences (i.e., procedural, conceptual, or mixed), and then they listed important factors influencing their preferences. Participants perceived procedural help as being contextualized, efficient, and easy to learn. Similarly, a few responses mentioned conceptual help as facilitating exploratory research and easy to learn. There are a considerable number of participants $(8,17.8 \%)$ with mixed preferences. In addition to personal preference, those participants listed contextualized help, ease of learning, efficiency, one-time versus repeated needs, and time limit as the influencing factors of their preferences.

\section{[Insert Table 5 about here.]}

Question 5 asked participants' preferred channel for help information and the distribution of responses is shown in Table 6. The most preferred channel is a Wiki or help website that allows users to search and read relevant information. Online chat or text messaging, face-to-face help, and email were also frequently mentioned in the responses. Other channels mentioned in a few responses include FAQs, Wikipedia, asking a professor or expert, phone call, and video tutorials.

[Insert Table 6 about here.]

The coding analysis revealed two connections among responses of the same participants: (1) preference of procedural help and visiting static help website without 
interactive features (15 participants); and (2) preference of visiting help website and avoiding direct communication with other people (7 participants). The first connection can be illustrated by the following comments from the same participant:

- "I prefer it (library help) to be more step-by-step so I can solve the immediate problem, but I like that to be contextualized within the framework of the site as a whole."

- "I prefer something static when searching for help information."

For the second connection, the tendency of avoiding direct help from other people could be due to the effort needed to form a clear description of the difficulty and the task goal, which may create additional pressure for the participant. This can be demonstrated by the following comments from another participant:

- "I only use help for technical questions, so I prefer it to be procedural. I just want to know how to get from Point A to Point B in the library system."

- "I prefer to get help from a comprehensive, static website. I like to solve my problems without the involvement of other people. ... It's a little uncomfortable to talk to someone I don't know over chat or phone, even if it's more efficient."

Furthermore, there are several common requirements of library help information across the responses: responsive to users' information seeking needs; easy to access; comprehensive (e.g., help information that guides users to a subject area); contextual to current problem or needs (e.g., help information embedded in search interface and results list); participatory (e.g., crowdsourced help like Quora and StackOverflow); and efficient for finding answers. 


\section{Discussion and Conclusion}

In this survey study, we measured participants' current help seeking preferences, expectations of future library help, acceptance of online help, preferences of help format (procedural versus conceptual), and the use of different help delivery channels. When seeking help about library resources, participants were divided into distinct groups, including: asking the library help desk, independent experimentation, searching the library website, and asking an instructor or peer. Participants carried this preference across questions, and prioritized help channels according to their help-seeking preferences.

Many participants would consider the library help desk and online help as the last resort after experimenting on their own. The availability of knowledgeable librarians or subject experts was considered the most important factor, even when an online help could help participants in their tasks (Question 2), which shows the importance of librarians' contribution to the creation of library help including both one-and-one references and online help content. If online help was developed to meet respondents' requirements on efficiency, convenient access and context relevancy, it would be used by the majority of participants.

Near half of the respondents preferred procedural or step-by-step help information (e.g., how to do a search within an e-book), while the other half either preferred help with better conceptual explanation (e.g., how the table of contents and index can help navigate within an e-book) or had mixed preferences between procedural and conceptual help. In addition, factors such as time limit and frequency of the obstacle could affect the choice of procedural versus conceptual help. Online help website or Wiki was considered the top 
choice for participants to read help information (Question 5). Other important channels mentioned in the responses include online chat or text messaging, face-to-face communication, and email. Although the order of different options in Question 5's responses does not completely match the responses to Question 1, both show that most respondents have established working strategies of incorporating experimentation, online help, and direct interaction with the library reference desk.

Many of the survey findings reflect the general patterns reported by previous studies on technical help documentation and library help features. More importantly, the survey results show the unique challenge of creating online help for libraries, as users have a dynamic range of help seeking preferences and mixed expectations of help content (e.g., procedural or conceptual) depending on the context. The survey participants showed a high degree of reliance on librarians and subject experts, even when online help could potentially solve their problems. This reliance on subject experts suggests that librarian support needs to be integrated with online help. Librarians and other subject experts would be able to understand the problem context, help users correctly identify the source of a problem, and lead users to the online help content with appropriate level of explanation and presentation format. As libraries keep expanding their scholarly resources, it is important to design an integrated, cross-channel help experience that involves both librarian touch points and the online help content.

In summary, we have identified users' preferences, attitudes, and expectations towards library help channels and online help from the survey. We expect the survey results to better inform improvement of existing help content, and to provide opportunities to expand help content in various contexts and formats. A limitation of the 
study is that we provided examples or prompts for some of the open-ended questions.

The wording of survey questions could be improved so that they are neutral and do not suggest possible answers for participants. Even though the survey encouraged participants to provide additional comments after the given questions, participants may not be motivated to write down other related thoughts. This was a trade-off we made in order to collect more responses in a relatively short time. We will plan a follow-up study with interviews and observations of users using library resources and librarians helping users solve problems. We expect the interviews and observations to validate the survey results, and more importantly, to map the detailed process of users interacting with librarians and the online help.

\section{References}

Craig, C.L. \& Friehs, C.G., 2013. Video and HTML: Testing Online Tutorial Formats with Biology Students. Journal of Web Librarianship, 7(3), pp.292-304.

Du, J., Xu, J. \& Fan, X., 2015. Help seeking in online collaborative groupwork: a multilevel analysis. Technology, Pedagogy and Education, 24(3), pp.321-337. Available at: http://search.proquest.com/docview/1698869356?accountid=13360.

Fisher, J., 1983. New Directions in Helping: Recipient reactions to aid, Elsevier.

Foster, N.F. \& Gibbons, S.L., 2007. Studying students: The undergraduate research project at the University of Rochester, Chicago, IL: Association of College \& Research Libraries.

Granfield, D. \& Robertson, M., 2008. Preference for reference: new options and choices for academic library users. Reference \& User Services Quarterly, 48(1), pp.44-53.

Ismail, L., 2013. Closing the Gap: Determining the Library Help-Seeking Preferences of Adult Learners in a Graduate Social Work Program. Reference \& User Services Quarterly, 53(2), pp.164-173. Available at: http://search.proquest.com/docview/1512201290?accountid=13360.

Ismail, L., 2010. What net generation students really want: Determining library helpseeking preferences of undergraduates. Reference Services Review, 38(1), pp.10-27.

Luo, L., 2014. Text a librarian: a look from the user perspective. Reference Services Review, 42(1), pp.34-51. Available at: 
http://search.proquest.com/docview/1507675384?accountid=13360.

Mestre, L.S., 2012. Student preference for tutorial design: a usability study. Reference Services Review, 40(2), pp.258-276.

Novick, D.G., Elizalde, E. \& Bean, N., 2007. Toward a more accurate view of when and how people seek help with computer applications. In Proceedings of the 25th annual ACM international conference on Design of communication - SIGDOC '07. New York, New York, USA: ACM Press, p. 95.

Novick, D.G. \& Ward, K., 2006. What users say they want in documentation. In Proceedings of the 24th annual conference on Design of communication SIGDOC '06. New York, New York, USA: ACM Press, pp. 84-91.

Pellegrino, C., 2012. Does Telling Them to Ask for Help Work?: Investigating Library Help-Seeking Behaviors in College Undergraduates. Reference \& User Services Quarterly, 51(3), pp.272-277. Available at: http://search.proquest.com/docview/963550699?accountid=13360.

Purchase, H.C. \& Worrill, J., 2002. An empirical study of on-line help design: features and principles. International Journal of Human-Computer Studies, 56(5), pp.539566.

Springshare, Libguides Community Site. http://libguides.com/.

Trenner, L., 1989. A comparative survey of the friendliness of online "help" in interactive information retrieval systems. Information Processing \& Management, 25(2), pp.119-136.

Tsai, C.-W., Shen, P.-D. \& Fan, Y.-T., 2015. Investigation of Student Learning Assistance through Online Academic Help-Seeking and a Mobile Application: A Quasi-Experimental Approach. International Journal of E-Adoption, 7(1), pp.1-16. Available at: http://search.proquest.com/docview/1689292109? accountid=13360.

Xie, H. (Iris), 2007. Help features in digital libraries: types, formats, presentation styles, and problems. Online Information Review, 31(6), pp.861-880.

Xie, H.I. \& Cool, C., 2007. Toward a better understanding of help seeking behavior: An evaluation of help mechanisms in two IR systems. Proceedings of the American Society for Information Science and Technology, 43(1), pp.1-16. 